\title{
Caso 01/2006 - Insuficiência Cardíaca Progressiva em Homem de 44 Anos de Idade
}

\author{
Case 01/2006 - Progressive Heart Failure in a 44-year Old Man
}

Tiago Senra Garcia dos Santos, Ariane Vieira Scarlatelli Macedo, Paulo J. Moffa, Maria Clementina Giorgi, Léa Maria Macruz Ferreira Demarchi, Jussara Bianchi Castelli Instituto do Coração do Hospital das Clínicas - FMUSP - São Paulo, SP

Homem de 44 anos de idade procurou atendimento médico por piora da dispnéia e edema de membros inferiores.

Aos 41 anos de idade sofreu episódio diagnosticado como pneumonia. Depois desse quadro, evoluiu com dispnéia desencadeada por esforços progressivamente menores até que, 2 anos após, esta ocorria aos esforços médios e também em decúbito horizontal. Surgiu então edema de membros inferiores. 0 paciente foi tabagista.

Por ocasião da avaliação médica (11/07/2002), fazia uso de 0,25 mg de digoxina, $40 \mathrm{mg}$ de furosemida, 25 mg de espironolactona, 37,5 mg de captopril, $100 \mathrm{mg}$ de ácido acetilsalicílico, $1.200 \mathrm{mg}$ de $\mathrm{KCl}$ e $30 \mathrm{mg}$ de propatilnitrato diários.

O exame físico revelou estertores em bases pulmonares, a semiologia cardíaca revelou hiperfonese do componente pulmonar da segunda bulha e presença de terceira bulha. O fígado foi palpado a $1 \mathrm{~cm}$ do rebordo costal e havia edema $++/ 4$ de membros inferiores.

O eletrocardiograma de 5/07/2002 (fig. 1) revelou ritmo sinusal com freqüência de 105 bpm, duração de $P$ 120 mseg, PR 185 mseg, QRS 84 mseg; SÂQRS + 120 paralelo, sobrecarga atrial direita e esquerda, sobrecarga ventricular direita do tipo sistólica, isto é, QRS estreito, $\mathrm{R}$ puro em $\mathrm{V}_{1}$ e infradesnivelamento de segmento ST parede ântero-septal .

A radiografia de tórax (5/07/2002) revelou espessamento pleural à direita com velamento de seio costofrênico direito, estrias em base pulmonar direita e aumento da área cardíaca.

A avaliação laboratorial (5/07/2002) revelou hemoglobina $17,1 \mathrm{~g} / \mathrm{dl}$; hematócrito 51\%, VCM 82 micra $^{3}$, leucócitos $8.900 / \mathrm{mm}^{3}$, plaquetas 184.000/ $\mathrm{mm}^{3}$, creatinina $1,3 \mathrm{mg} / \mathrm{dl}$, glicose $96 \mathrm{mg} / \mathrm{dl}$, potássio 5,2 mEq/l, sódio $138 \mathrm{mEq} / \mathrm{l}$. As reações sorológicas para doença de Chagas foram negativas; a pesquisa do anticoagulante lúpico foi negativa. O coagulograma foi normal, a atividade de fator $\mathrm{V} 72 \%$, de antitrombina III $79 \%$ e de proteína C 92\%. A pesquisa de dímero D foi positiva. Os anticorpos anticardiolipina das classes IgM

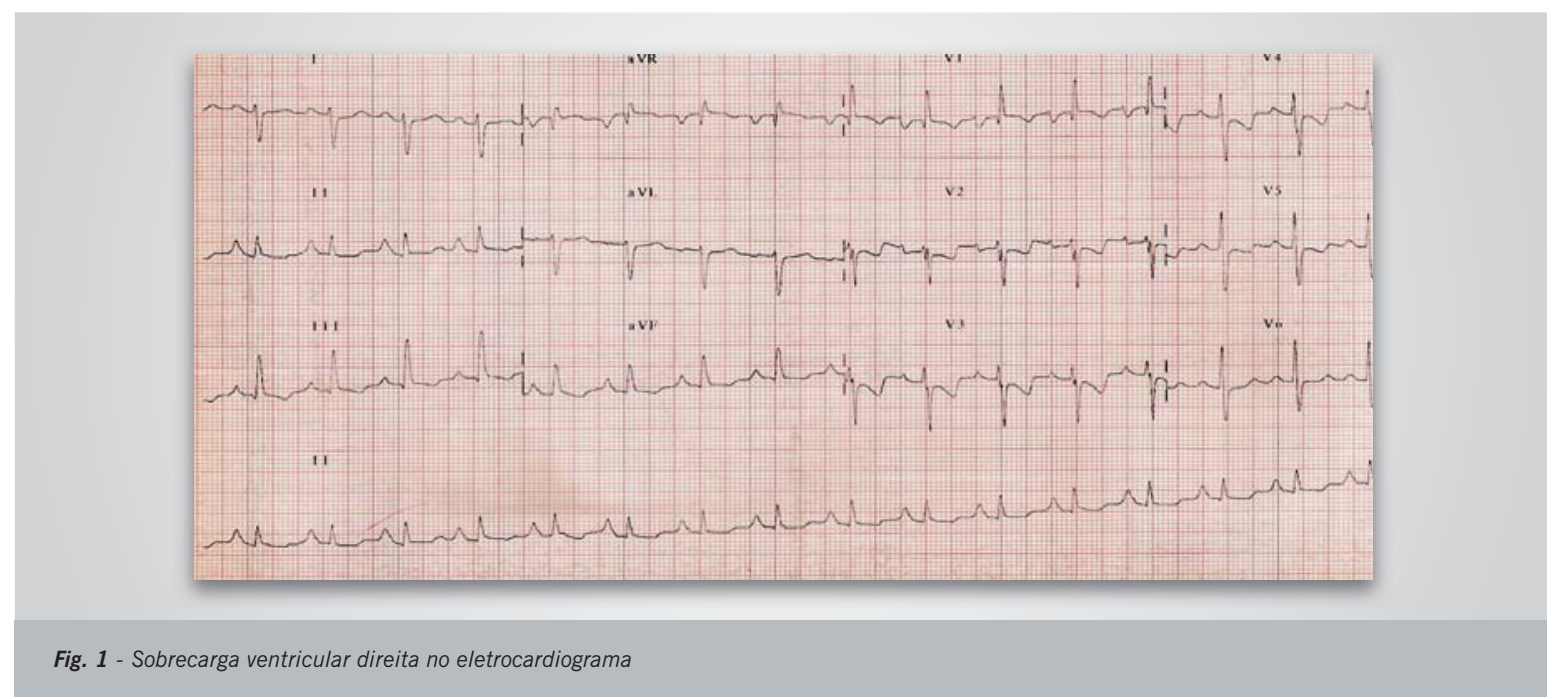

Editor da Seção: Alfredo José Mansur (ajmansur@incor.usp.br) Editores Associados: Desidério Favarato (dclfavarato@incor.usp.br) Vera Demarchi Aiello (anpvera@incor.usp.br)

Correspondência: Jussara Bianchi Castelli • InCor - Av. Dr. Enéas de Carvalho Aguiar, 44 - $05403-000$ - São Paulo, SP E-mail: anpjussara@incor.usp.br 
e IgG foram normais. O colesterol total foi $188 \mathrm{mg} / \mathrm{dl}$, o HDL-colesterol $31 \mathrm{mg} / \mathrm{dl}$, o LDL-colesterol $132 \mathrm{mg} / \mathrm{dl}$, os triglicérides $126 \mathrm{mg} / \mathrm{dl}$, a proteína C reativa $43 \mathrm{mg} / \mathrm{l}$. Foram detectadas larvas de Strongyloides stercoralis no exame proctoparasitológico.

O ecocardiograma (15/07/2002) revelou hipertensão na artéria pulmonar, dilatação e hipocinesia de ventrículo direito, insuficiência tricúspide moderada e imagens sugestivas de grandes trombos em átrio direito, ventrículo direito (pedunculado), tronco pulmonar. O ventrículo esquerdo foi considerado normal (tab. 1).

A cintilografia pulmonar inalatória e perfusional de 17/07/2002 (fig. 2) revelou ventilação presente e perfusão diminuída em lobo superior direito, lobo médio e segmento ântero-basal do lobo inferior direito, e segmentos anterior, ápico-posterior do lobo superior, língula e lateral basal do lobo inferior esquerdo. Os achados foram considerados altamente sugestivos de tromboembolismo pulmonar.

Vísceras abdominais e vasos foram considerados normais na ultra-sonografia abdominal.

A ultra-sonografia dos vasos dos membros inferiores (17/07/2002) revelou extensa trombose venosa de ilíacas externas e trecho femoropoplíteo em ambos os membros, com sinais de recanalização à direita e oclusão poplítea esquerda.

A angioressonância magnética de artérias pulmonares (22/07/2002) revelou dilatação do tronco pulmonar e de seus ramos e presença de trombo em ramos pulmonares direito e esquerdo.

A ventriculografia por ressonância magnética revelou sobrecarga de câmaras direitas e trombos em parede ântero-lateral e via de saída de ventrículo direito.

Foram feitos os diagnósticos de tromboembolia pulmonar e cor pulmonale. Recebeu tratamento anticoagulante, inicialmente com heparina de baixo peso molecular, depois com warfarina; além de 25 mg de espironolactona, 37,5 $\mathrm{mg}$ de captopril e $40 \mathrm{mg}$ de furosemida.

Houve melhora dos sintomas e o paciente foi encaminhado para seguimento médico ambulatorial.

A tomografia de abdome foi normal e a tomografia helicoidal de tórax (12/03/2003) revelou dilatação do tronco pulmonar (42 mm) e de seus ramos e falhas de enchimento nos principais ramos direitos e esquerdos e na artéria lobar inferior esquerda, sugestiva de tromboembolia pulmonar.

O controle ecocardiográfico (24/03/2003) não revelou alteração em relação ao exame anterior, a não ser pelo desaparecimento dos sinais de trombose intracavitária e artéria pulmonar (tab. 1).

A arteriografia pulmonar (jul/2003) revelou dilatação do tronco da artéria pulmonar e do ramo esquerdo; havia diminuição do calibre distal do ramo pulmonar direito, que foi interpretada como possivelmente secundária à trombose.

\begin{tabular}{|ccc|}
\hline \multicolumn{3}{|c|}{ Tabela 1 - Ecocardiogramas } \\
\hline & 15 jul 02 & 24 mar 03 \\
\hline Septo interventricular (mm) & 8 & 9 \\
\hline Parede posterior (mm) & 8 & 9 \\
\hline Diâmetro diastólico VE (mm) & 44 & 44 \\
\hline Diâmetro sistólico VE (mm) & 29 & 25 \\
\hline Aorta (mm) & 30 & 30 \\
\hline Átrio esquerdo (mm) & 32 & 35 \\
\hline Ventrículo direito (mm) & 40 & 40 \\
\hline Motilidade do ventrículo direito & Hipocinesia acentuada & Hipocinesia acentuada \\
\hline Pressão sistólica de VD (mmHg) & 64 & 65 \\
\hline Tronco pulmonar (mm) & - & 36 \\
\hline Trombos & AD; VD; TP & Não \\
\hline VE- ventrículo esquerdo; VD- ventrículo direito; AD- átrio direito; \\
\hline TP- tronco pulmonar
\end{tabular}

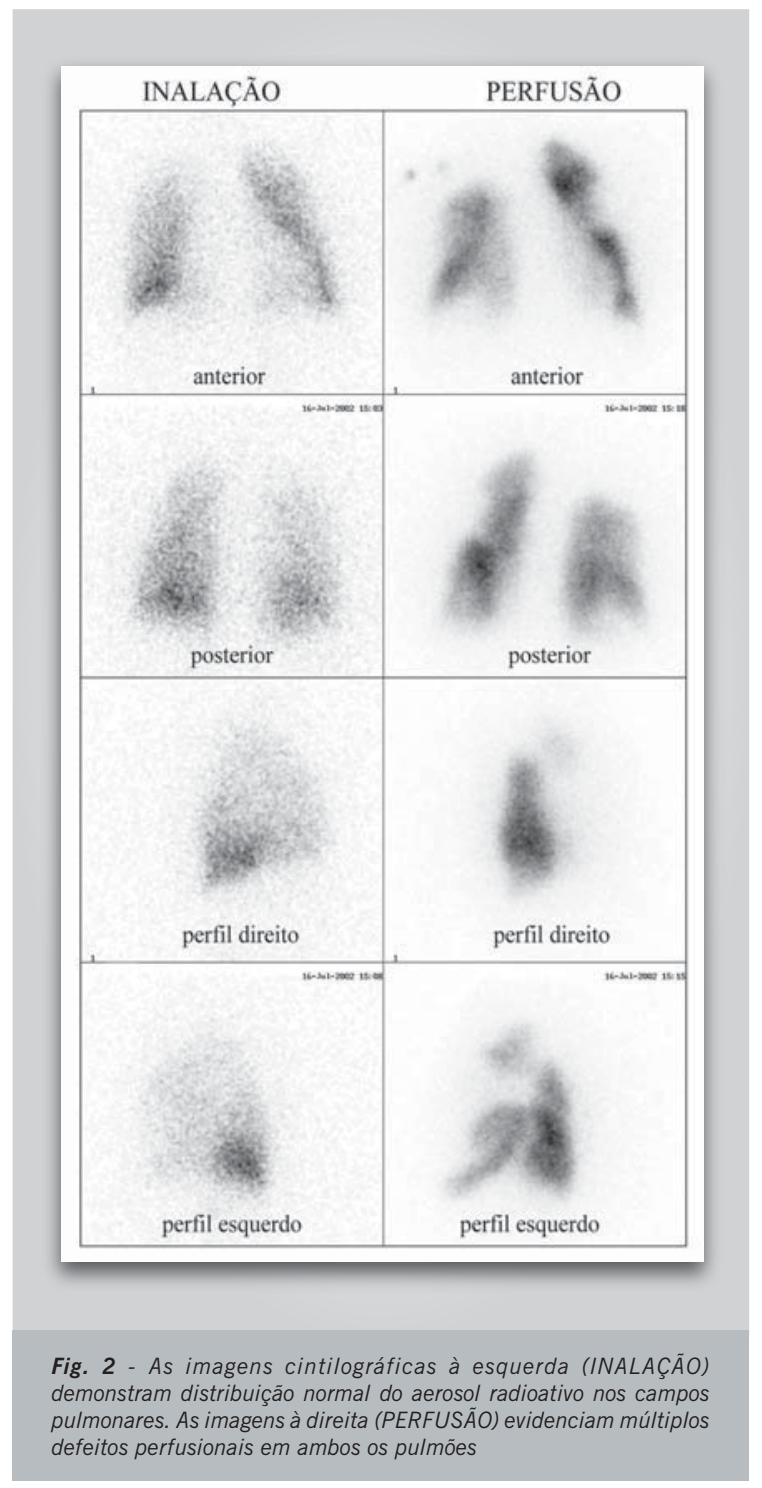


As pressões da artéria pulmonar (sistólica/diastólica/ média) foram 73/32/50 mmHg e a pressão média do átrio direito foi $22 \mathrm{mmHg}$.

O paciente evoluiu com cansaço aos esforços habituais apesar da medicação prescrita até agosto de 2003 quando apresentou piora da dispnéia, agora aos pequenos esforços, reaparecimento de edema e episódio de présíncope. Foi re-hospitalizado.

O exame físico (15/08/2003) revelou pressão arterial 140/80 mmHg, freqüência cardíaca 80 bpm, ausculta pulmonar normal, hiperfonese de segunda bulha em área pulmonar e edema $++++/ 4$ de membros inferiores .

Os exames laboratoriais (15/08/2003) revelaram hemoglobina $17,3 \mathrm{~g} / \mathrm{dl}$, hematócrito $51 \%$, VCM $77 \mu \mathrm{m}^{3}$, 8.200 leucócitos $/ \mathrm{mm}^{3}$, plaquetas $112.000 / \mathrm{mm}^{3}$, sódio $138 \mathrm{mEq} / \mathrm{l}$, potássio 4,3 mEq/l, INR 1,97, relação de tempos TTPA 2,5, uréia $54 \mathrm{mg} / \mathrm{dl}$, creatinina 1,5 mg/dl.

Dois dias depois houve piora da dispnéia que se tornou intensa e o paciente apresentou cianose leve. Dez dias após apresentou choque e necessitou de uso de aminas vasoativas.

A avaliação laboratorial evolutiva (set/2003) revelou INR 2,5, relação de TTPA 1,5, hemoglobina 15,1 g/dl, hematócrito $44 \%$, leucócitos $10.800 / \mathrm{mm}^{3}$, plaquetas $78.000 \mathrm{~mm}^{3}$, creatinina $1,7 \mathrm{mg} / \mathrm{dl}$; uréia $89 \mathrm{mg} / \mathrm{dl}$. Houve piora da dispnéia e o paciente sofreu parada cardíaca (4/09/2003).

\section{DESCRIÇÃO DO ELETROCARDIOGRAMA}

O ritmo é sinusal, a freqüência cardíaca 100 batimentos por minuto, intervalo PR 200 mseg, duração da onda P $100 \mathrm{mseg}$, do QRS $80 \mathrm{mseg}$, do QT 320 mseg. $O$ eixo de QRS está a $120^{\circ}$. A onda P apresenta amplitude de $3 \mathrm{~mm}$ em II, é negativa em V1 e isoelétrica em V2 e positiva em V3 com área negativa em V1 com duração de 40 mseg.

Há desvio do SÂQRS para a direita, e qR com padrão de "strain" em V1 e RS em V5 e V6. Há infradesnível do segmento ST em V2 e V3 e ondas T negativas e V1 a $\mathrm{V} 5$ e derivações inferiores. Os achados permitem 0 diagnóstico de sobrecarga ventricular direita e sobrecarga atrial direita, associada a alterações do segmento ST e onda T. O aspecto de sobrecarga atrial esquerda em V1 (pseudo Morris) é justificado pela rotação horária do coração e deslocamento do átrio esquerdo para trás e anteriorização do átrio direito.

(Dr. Paulo J. Moffa)

\section{Cintilografia de inalação e PERFUSÃO PULMONARES}

Cintilografia pulmonar inalatória foi realizada com aerosol de ácido dietileno triamino pentaacético marcado com tecnécio-99m nas incidências anterior, posterior, laterais direita e esquerda e oblíquas posteriores direita e esquerda de tórax. Evidencio-se aeração presente em ambos os campos pulmonares. As imagens de perfusão pulmonar foram realizadas nas mesmas incidências imediatamente após a administração venosa de macroagregado de albumina marcado com tecnécio$99 \mathrm{~m}$. Observou-se hipoperfusão em projeção do lobo superior direito, lobo médio direito, segmento anterior basal do lobo inferior direito; segmentos anterior e parte do ápico-posterior do lobo superior esquerdo, parte da língula e segmento lateral basal do lobo inferior esquerdo. As alterações referidas são altamente sugestivas de tromboembolismo pulmonar.

(Dra. Maria Clementina Giorgi)

\section{Aspectos Clínicos}

O caso em questão refere-se a um homem de 44 anos de idade com sintomas e sinais de insuficiência cardíaca, submetido a extensa investigação diagnóstica que revelou quadro de cor pulmonale secundário a tromboembolismo pulmonar (TEP). Recebeu tratamento clínico com melhora pelo período de um ano, quando voltou a apresentar os mesmos sintomas, sendo internado. Evoluiu nos 20 dias seguintes com piora progressiva do quadro, instabilidade hemodinâmica e óbito.

A condução do caso em questão seguiu de maneira geral as diretrizes internacionais e brasileiras de investigação de hipertensão pulmonar $(\mathrm{HP})^{1,2}$.

A anamnese e exame físico iniciais sugeriam a presença de insuficiência cardíaca direita e hipertensão pulmonar, hipóteses que foram reforçadas pelos achados eletrocardiográficos e da radiografia de tórax.

Tendo em vista a definição de cor pulmonale da Organização Mundial da Saúde - alteração da estrutura e/ou função do ventrículo direito resultante de doenças do pulmão ou sua vasculatura, exceto quando estas doenças são resultado de alterações primariamente do lado esquerdo do coração e nas cardiopatias congênitas - os achados do ecocardiograma neste paciente foram fundamentais para o estabelecimento do diagnóstico, uma vez que descartaram disfunção ventricular esquerda e anomalias congênitas.

De fato, a literatura mostra que critérios clínicos, eletrocardiográficos e de radiografia de tórax podem identificar em conjunto 39\% dos pacientes com cor pulmonale, enquanto o ecocardiograma chega a diagnosticar $75 \%$ dos casos $^{3}$. 0 achado de insuficiência tricúspide está presente em $80 \%$ dos pacientes com pressão sistólica de artéria pulmonar (PSAP) superior a $35 \mathrm{mmHg}$ e em $95 \%$ dos pacientes com PSAP maior que $50 \mathrm{mmHg}$, como encontrado no paciente em questão ${ }^{4}$. Outros possíveis achados ausentes neste caso são o abaulamento do septo interventricular para a esquerda, ventrículo esquerdo diminuído e função diastólica esquerda comprometida. 
Uma vez estabelecido o diagnostico de HP faz-se necessário o esclarecimento de sua etiologia. Apesar do achado neste caso de imagens sugestivas de grandes trombos em átrio direito, ventrículo direito e tronco pulmonar já ser compatível com tromboembolismo pulmonar crônico (TEPC), uma vasta gama de outras etiologias já foi relacionada à HP e deve ser relembrada.

Doenças que causam vasoconstrição hipóxica - doença pulmonar obstrutiva crônica (DPOC), apnéia obstrutiva do sono, obesidade, doenças neuromusculares, anormalidades torácicas - têm importante papel na etiologia da HP. Destaca-se a doença pulmonar obstrutiva crônica, responsável pela maioria dos casos. Nesse contexto, teria sido útil a realização de prova de função pulmonar no caso em questão, tendo em vista o passado de tabagismo do paciente. A própria HP pode causar padrão restritivo leve em até $20 \%$ dos pacientes. Outra utilidade deste exame teria sido a avaliação de eventual contra-indicação a tromboendarterectomia, como veremos mais tarde.

As doenças que acometem o leito arterial pulmonar são várias: TEPC, neoplasias, hipertensão pulmonar primária, esquistossomose, hemoglobinopatias, doenças do colágeno, infecção pelo HIV, uso de drogas anorexígenas, entre outras.

Naqueles pacientes em que o ecocardiograma não é tão sugestivo de uma etiologia como no caso presente, a cintilografia inalatória e perfusional tem papel divisor de águas neste ponto da investigação da $\mathrm{HP}^{5}$.

Pacientes com TEPC apresentam um ou mais defeitos de perfusão segmentares ou ainda maiores, enquanto que a perfusão está normal ou apresenta defeitos subsegmentares nos pacientes com HP primária. Vale ressaltar que a magnitude dos defeitos de perfusão freqüentemente subestima o grau de obstrução vascular pulmonar em vista do complexo processo de recanalização ${ }^{6}$. A cintilografia também não distingue 0 TEPC de outras causas de obstrução luminal, como o sarcoma de artéria pulmonar, vasculites, compressão vascular secundária a adenopatia ou fibrose mediastinal e pode ter resultado inconclusivo nos pacientes com disfunção ventricular.

Tendo em vista os achados do ecocardiograma e ainda seguindo as diretrizes internacionais e brasileiras, procedeu-se à cintilografia inalatória e perfusional, que reforçou ainda mais a hipótese de tromboembolismo pulmonar neste caso.

Tomando-se os resultados dos exames já mencionados e os achados da ressonância magnética de artérias pulmonares e da ultra-sonografia dos vasos dos membros inferiores foi feito o diagnóstico de tromboembolia pulmonar e cor pulmonale.

Há alguns anos acreditava-se que a incidência de TEPC não ultrapassasse $0,5 \%$ dos pacientes sobreviventes de um episódio de tromboembolismo pulmonar ${ }^{7}$. No entanto, estudos mais recentes mostram que até 3,8\% dos pacientes com TEP evoluem com hipertensão pulmonar secundária a TEPC nos dois anos seguintes ao evento, apesar de uso de anticoagulação por período prolongado ${ }^{8}$. Dentre os fatores de risco para tal evolução destacaram-se múltiplos episódios de TEP, grande defeito de perfusão, idade menor que 50 anos e apresentação inicial de TEP idiopática. Vale ressaltar que parte dos pacientes com TEP nunca são diagnosticados - a incidência de hipertensão pulmonar em pacientes com TEP não-tratado é desconhecida.

A maior parte dos pacientes com HP e TEPC só procuram atendimento tardiamente no curso da doença, provavelmente devido à grande reserva do leito vascular pulmonar e ao não-reconhecimento do primeiro episódio embólico, fato que pode ter ocorrido no caso em questão.

O encontro de trombose venosa profunda (TVP), como neste caso, varia de 35 a $70 \%$ dos $\operatorname{casos}^{9}$; o risco de embolia pulmonar aumenta quanto mais proximal for a trombose.

Atualmente, um fator de risco para trombose, seja ele congênita ou adquirida, pode ser encontrado em até $80 \%$ dos pacientes, sendo que, em geral, há mais de um fator de risco envolvido ${ }^{10}$.

Uma tendência genética para fenômenos tromboembólicos geralmente está presente em indivíduos jovens (menores que 50 anos) vítimas de TVP/TEP, principalmente nos casos recorrentes, podendo ser identificada, em algumas séries, em 24 a 37\% dos pacientes com TVP. As mutações do fator $V$ de Leiden e do gene da protrombina podem ser responsabilizadas por até $60 \%$ dos casos, sendo o remanescente dos casos atribuído principalmente às deficiências de proteínas anticoagulantes como proteína S, C e antitrombina III ${ }^{11}$. Outros distúrbios da coagulação como disfibrinogenemias, deficiência do cofator II da heparina, deficiência do fator XII e aumento da atividade do fator VIII também podem ser elencados como possíveis causas de trombofilias herdadas. Atualmente, considera-se que a elevação nos níveis séricos de fator VIII é um marcador independente do aumento do risco de trombose, principalmente em negros e em usuárias de contraceptivos orais. Este distúrbio também se associa a fenômenos tromboembólicos recorrentes ${ }^{12}$ e é encontrado em mais de $40 \%$ dos pacientes com TEPC ${ }^{13}$.

Dentre os diversos fatores trombogênicos adquiridos podemos enumerar as doenças malignas, a síndrome do anticorpo antifosfolípide e a hiper-homocisteinemia. Cirurgias prévias, tabagismo, doenças mieloproliferativas, trauma, imobilização, gravidez e uso de contraceptivos orais são outros fatores que devem ser lembrados.

Pacientes com câncer têm um estado de hipercoagulabilidade em razão da produção de substâncias com atividade pró-coagulante. Os tumores associados a tromboembolismo são clinicamente evidentes por ocasião do evento em até $78 \%$ dos casos, porém o tromboembolismo pode preceder o diagnóstico do 
câncer e este se manifestar anos após o primeiro episódio, principalmente nos casos de trombose venosa recorrente ${ }^{14}$. Os tumores mais associados com tromboembolismo são os de pulmão, pâncreas, cólon, rim e próstata. Nesse paciente, a investigação de neoplasias de pulmão, pâncreas e rim foi realizada através de tomografia computadorizada de tórax e abdome, que foram negativas. Não foram investigados cólon e próstata.

Ainda no cenário das trombofilias adquiridas, destacamse a hiper-homocisteinemia e a síndrome do anticorpo antifosfolípide. Evidências crescentes mostram que a hiper-homocisteinemia, além de ser um fator de risco para aterosclerose, associa-se também a um aumento no risco de TVP/TEP ${ }^{15}$. Estudos recentes têm mostrado uma taxa até 3 vezes maior de fenômenos tromboembólicos em pacientes com aumento dos níveis séricos de homocisteína. A trombose venosa é a manifestação inicial mais freqüente da síndrome do anticorpo antifosfolípide e correlaciona-se à presença do anticorpo anticardiolipina, ocorrendo em até $32 \%$ dos seus portadores e podendo ser detectada em até $21 \%$ dos pacientes vítimas de TVP ${ }^{16}$. Associa-se a lúpus eritematoso sistêmico (LES) e outras doenças reumatológicas, sendo que a incidência de trombose é maior quando LES está presente. No paciente em questão foi feita uma ampla investigação para trombofilias tanto adquiridas como congênitas, sem, no entanto, esclarecimento diagnóstico. Entretanto, a hipótese de trombofilia não pode ser excluída visto que causas freqüentes como hiper-homocisteinemia, mutação do gene da protrombina e aumento dos níveis séricos de fator VIII não foram pesquisados.

A progressão da HP parece estar mais relacionada a remodelamento da arquitetura vascular pulmonar que a novos eventos embólicos ou trombose nas artérias pulmonares. Esta hipótese apóia-se em vários achados: baixa correlação entre 0 grau de obstrução proximal e 0 grau de HP, piora hemodinâmica na ausência de eventos embólicos documentados e alterações histopatológicas em vasos relacionados ou não às obstruções.

O prognóstico dos doentes não tratados é sombrio e parece estar relacionado ao grau de HP: a sobrevida em 5 anos aproxima-se de $30 \%$ nos pacientes com PAP média maior que $40 \mathrm{mmHg}$ e $10 \%$ naqueles com PAP média superior a $50 \mathrm{mmHg}^{17}$.

O paciente em questão iniciou tratamento clínico com varfarina, espironolactona, captopril e furosemida e seguiu em acompanhamento ambulatorial.

O tratamento clínico na HP secundária a TEPC é visto como ponte para o tratamento cirúrgico definitivo. Somente recebem tratamento clínico exclusivo aqueles pacientes não-candidatos à cirurgia.

A anticoagulação, o uso de diuréticos e vasodilatodores são recursos terapêuticos clássicos na HP.

A anticoagulação com varfarina neste caso é inquestionável, faltando ainda consenso na literatura quanto ao INR ótimo.

0 uso de diuréticos visa diminuir a pré-carga do ventrículo direito, otimizar o mecanismo de FrankStarling e melhora hemodinâmica. Há no entanto risco de depleção excessiva do volume intravascular, com conseqüente hemoconcentração, piora do débito cardíaco, síncope e piora da função renal - como apresentado pelo paciente no retorno ao hospital.

O uso de vasodilatadores - especialmente os bloqueadores de canal de cálcio - está consagrado naqueles pacientes que apresentam teste invasivo de vasodilatação pulmonar: queda da PAP em $10 \mathrm{mmHg}$, redução da PAP média e/ou resistência vascular pulmonar em $20 \%$ ou mais, ou diminuição de 30\% tanto na PAP quanto na resistência, dependendo da instituição. Seu uso no contexto clínico do paciente em questão não está definido.

A indicação de oxigenioterapia está bem estabelecida nos pacientes com HP secundária a DPOC ${ }^{18}$. No entanto, seu uso em outros grupos de pacientes com HP reduziu agudamente a PAP média e a resistência vascular pulmonar e aumentou o débito cardíaco ${ }^{19}$. Ainda faltam estudos do benefício a longo prazo dessa terapia.

Nos últimos anos, novas classes de drogas utilizadas na HP primária e secundária a outras etiologias têm sido testadas em pequenos ensaios clínicos envolvendo pacientes com HP secundária a TEPC.

O uso do epoprostenol, um análogo de prostaciclina de administração endovenosa central contínua, associouse com uma queda de $28 \%$ na resistência vascular pulmonar e de $66 \%$ nos níveis de $\mathrm{BNP}^{20}$. A administração de iloprost, outro análogo de prostaciclina inalatório, apresentou resultados potencialmente promissores na literatura até o momento ${ }^{21}$.

O uso do óxido nítrico neste tipo de paciente ainda se restringe ao período pós-operatório em relatos de caso na literatura.

Outras drogas como a I-arginina (precursor do óxido nítrico), o sildenafil (inibidor da fosfodiesterase), o bosentan (inibidor do receptor de endotelina-1), a almitrina bismesilato (droga estimulante do seio carotídeo), o treprostinil e o beraprost (análogos da prostaciclina) ainda não foram testadas no contexto do paciente em questão.

Tendo em vista a baixa taxa de sobrevida nos pacientes com HP grave secundária a TEPC tratados clinicamente, a busca por um tratamento efetivo fez crescer o interesse por métodos cirúrgicos.

A tromboendarterectomia consiste basicamente na retirada dos trombos durante parada circulatória e hipotermia profunda intermitentes, favorecendo a livre passagem do sangue pelo interior da artéria pulmonar com conseqüente queda da PAP. O procedimento tem sua indicação clássica nos pacientes sintomáticos que apresentam distúrbios ventilatórios e/ou hemodinâmicos 
em repouso ou com exercício e com resistência vascular pulmonar maior que $300 \mathrm{dyn}^{2}$. Outros determinantes críticos para a indicação cirúrgica são a localização e a extensão dos trombos, que devem se restringir à artéria pulmonar, aos seus ramos lobares ou às artérias segmentares proximais e a ausência de eventos tromboembólicos nos 3 meses prévios ao procedimento. Deve haver concordância entre a magnitude dos trombos e a resistência vascular pulmonar, uma vez que o achado de resistência alta com poucos trombos sugere doença distal inabordável. A única contra-indicação absoluta constante na literatura é a presença de doença pulmonar obstrutiva ou restritiva grave. Idade avançada, falência ventricular direita grave - como observado no paciente em questão - ou a presença de outras comorbidades aumentam o risco do procedimento, porém não são contra-indicações absolutas. Não há consenso entre os autores se a tromboendarterectomia deve ser precedida de colocação de filtro de veia cava. No Instituto do Coração-HCFMUSP este procedimento é realizado somente nos pacientes com TVP confirmada.

Uma coorte retrospectiva aqui realizada analisou 26 pacientes portadores de embolia pulmonar crônica submetidos a tromboendarterectomia e seguidos por aproximadamente por 3,5 $\operatorname{anos}^{22}$. A mortalidade no pós-operatorio imediato foi de $11,6 \%$; a análise da evolução tardia dos demais pacientes correspondeu a um período de 42 meses no qual houve apenas um óbito por novo TEP. A cintilografia pulmonar e a arteriografia revelaram redução do acometimento lobar pulmonar e o ecocardiograma mostrou significativa redução da PSAP e do diâmetro diastólico do ventrículo direito. Com relação ao comportamento da classe funcional, 92\% dos pacientes apresentavam classe funcional III ou IV no pré-operatorio, enquanto que no pós-operatorio $89 \%$ encontravam-se assintomáticos ou em classe I e o restante em classe funcional II.

Nos pacientes não-candidatos a tromboendarterectomia restam três opções terapêuticas: tratamento clínico, angioplastia de artéria pulmonar por cateter-balão e transplante de pulmão.

Neste caso, a tromboendarterectomia não foi um recurso terapêutico utilizado.

O histórico do doente e as características de sua evolução - piora do quadro respiratório, cianose, instabilidade hemodinâmica - apontam para uma causa cardíaca para o óbito.

Hipóteses diagnósticas - Assim, temos como hipóteses para a causa do óbito: 1) falência ventricular direita secundária a hipertensão pulmonar e tromboembolismo pulmonar crônico; 2) novo episódio de tromboembolismo pulmonar.

(Tiago Senra Garcia dos Santos, Ariane Vieira Scarlatelli Macedo)

\section{NeCropsia}

$\mathrm{Na}$ autópsia, foi comprovada a presença de tromboembolismo pulmonar crônico, com hipertensão pulmonar. $\mathrm{O}$ quadro era caracterizado pela presença de alterações arteriais e arteriolares obstrutivas em circulação pulmonar, recentes e antigas, a saber: trombose recente em organização em artérias pulmonares centrais ("a cavaleiro"), tromboembolias recentes em artérias intraparenquimatosas do pulmão esquerdo e sinais sugestivos de trombos antigos recanalizados em arteríolas pulmonares esparsas (fig. 3). O pulmão esquerdo sofreu extenso infarto hemorrágico, acometendo todo o lobo inferior. O coração apresentava aumento de tamanho, pesando 516 g, à custa de intensa dilatação e hipertrofia de câmaras cardíacas direitas (fig. 4), caracterizando o diagnóstico de cor pulmonale crônico e agudo. Achado incomum foi a presença de aneurisma na parede posterior do átrio direito (fig. 5), acima da desembocadura do seio coronário, medindo $3 \times 2 \times 1 \mathrm{~cm}$, preenchido por trombo em organização, cuja histologia mostrava a parede composta de tecido miocárdico com fibrose intersticial, sem sinais de infarto recente.

Nos demais órgãos foram encontradas alterações secundárias à insuficiência cardíaca congestiva principalmente direita, tais como edema subcutâneo generalizado, com dermatite ocre em membros inferiores e congestão passiva crônica hepática, com esplenomegalia. Trombose em organização foi detectada também em veia ilíaca direita (trombo com cerca de $5 \times 2 \mathrm{~cm}$ ) que possivelmente colaborou para o tromboembolismo crônico pulmonar, e em veia do sistema porta-hepático.

A causa imediata para o óbito foi atribuída a tromboembolia pulmonar difusa, com infarto pulmonar. Esta teve colaboração de baixo débito, evidenciado pelos seguintes sinais: infartos subendocárdicos, pequenos e múltiplos, em diferentes estádios de evolução, necrose

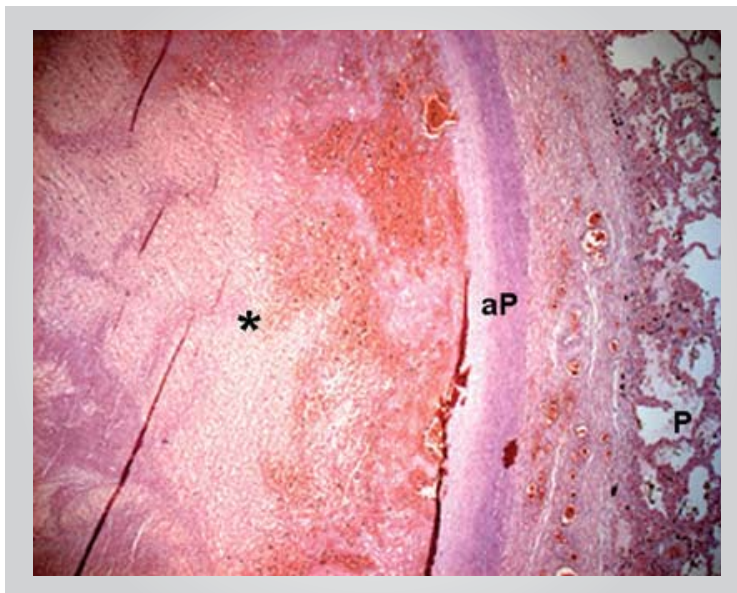

Fig. 3 - Fotomicrografia mostrando corte histológico de uma das artérias pulmonares hilares com trombose em organização. Trombos (*) aderidos à parede da artéria pulmonar $(a P)$ ocluem totalmente a luz. Do lado direito, o tecido esponjoso é o parênquima pulmonar (P) adjacente. (Corado pela hematoxilina \& eosina; objetiva $5 X$ ) 

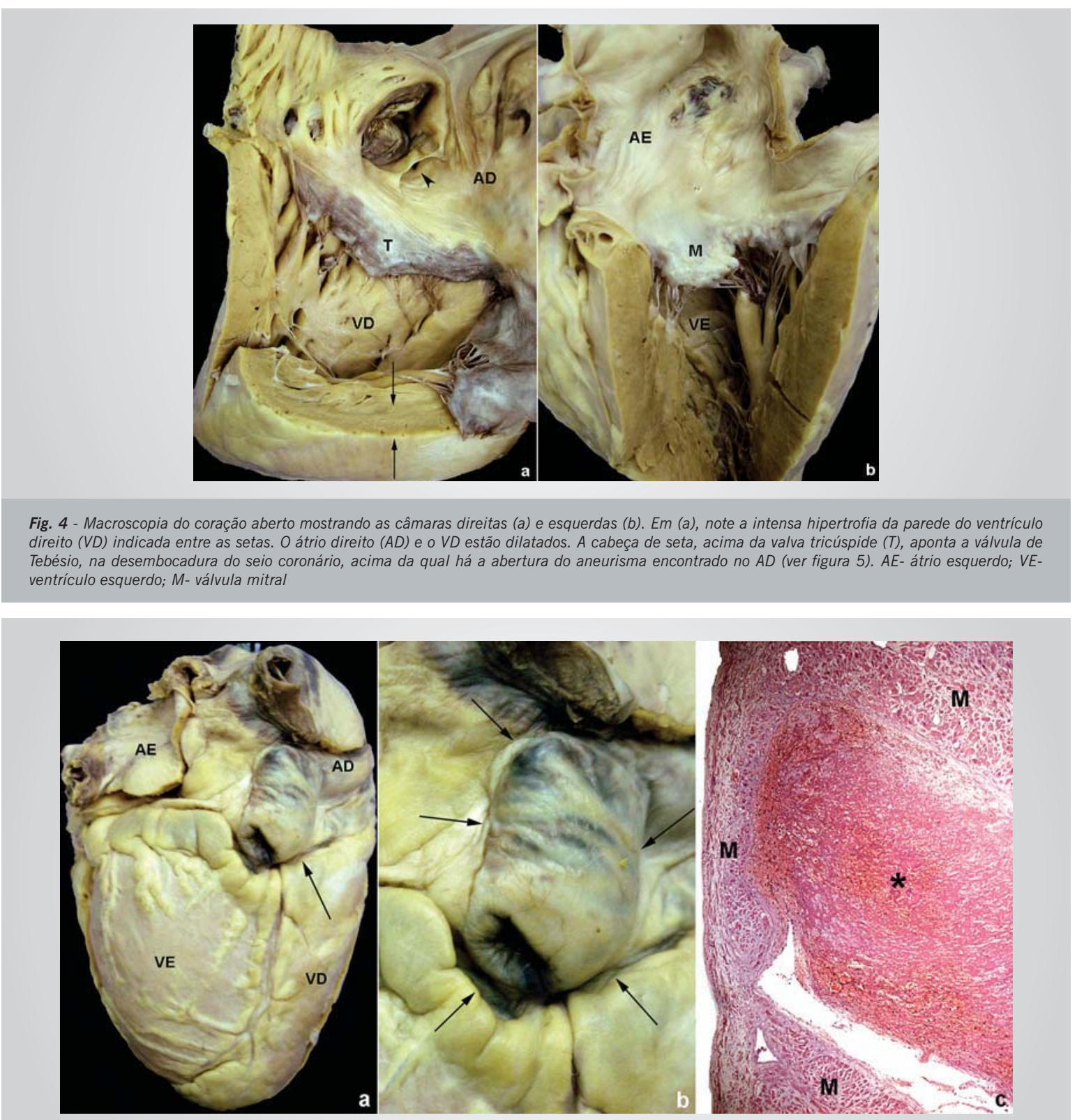

Fig. 5 - Macro e microscopia do aneurisma do átrio direito. Em (a), o coração é visto externa e posteriormente. A seta aponta a localização do aneurisma. Os ventrículos esquerdo (VE) e direito (VD), e os átrio esquerdo ( $A E$ ) e direito (AD) são indicados. (b) Detalhe macroscópico do aneurisma, saliência digitiforme na parede posterior do $A D$. A abertura interna do aneurisma é vista na figura 4. (c) Fotomicrografia da parede do aneurisma do $A D$, com a parede composta por miocárdio (M) e a luz preenchida por trombos (*) em organização

tubular aguda dos rins, edema agudo dos pulmões e necrose centro-lobular hepática.

Diagnósticos anatomopatológicos - Tromboembolismo pulmonar a cavaleiro em organização; tromboembolismo pulmonar crônico, com hipertensão pulmonar; cor pulmonale crônico e agudo, descompensado por infarto pulmonar extenso recente; aneurisma de átrio direito; tromboses venosas em veia ilíaca direita e do sistema porta-hepático.

(Jussara Bianchi Castelli, Léa Maria Macruz Ferreira Demarchi)

\section{Comentários}

Por definição, cor pulmonale é uma alteração na estrutura e função do ventrículo direito, cuja causa de base está no sistema respiratório, gerando aumento de pressão sangüínea na pequena circulação. Ou seja, invariavelmente a hipertensão pulmonar (HP) precede o cor pulmonale. Para falar de sua expressão morfológica, a sobrecarga de pressão resulta em paulatina hipertrofia cardíaca ventricular direita, ou o chamado cor pulmonale crônico. Cor pulmonale agudo se refere à dilatação do ventrículo direito por sobrecarga aguda, que no caso 
deve estar relacionada ao infarto recente e extenso em pulmão esquerdo.

Diferentes mecanismos patofisiológicos envolvendo o tecido pulmonar diretamente podem levar a vasculopatia, HP e, subseqüentemente, a cor pulmonale. 0 comprometimento pulmonar vascular mais importante pela freqüência é o do setor arterial. Os sitiados em veias (venopatia oclusiva) e em microcirculação (microvasculopatia pulmonar) são raros ${ }^{23}$. Na HP arterial, estes mecanismos patogenéticos incluem: vasoconstricção (por hipóxia ou acidemia); lesão anatômica do leito arterial secundária a desordens pulmonares tais como enfisema, DPOC, tromboembolismo pulmonar e doença pulmonar intersticial, entre outras; aumento da viscosidade sangüínea secundária a desordens hematológicas, por exemplo, policitemia vera, anemia falciforme, macroglobulinemia; e, hipertensão pulmonar primária idiopática. Outros estados freqüentes que levam a HP arterial e sobrecarga de ventrículo direito, tais como valvopatias e cardiopatias congênitas com desvio de sangue da esquerda para a direita, têm a anomalia de base no coração e assim, estão fora da definição de cor pulmonale.

A expressão anatômica da HP arterial comumente é representada por placas de ateroma nas artérias elásticas (correspondendo ao tronco pulmonar e boa parte das artérias pulmonares centrais), hipertrofia da média em artérias elásticas e musculares, proliferação intimal em artérias musculares, muscularização de arteríolas e formas distintas de remodelamento do vaso, tais como fibrose laminar, concêntrica ou excêntrica, lesão plexiforme (lesão vascular que lembra um tufo glomerular), por vezes com trombose in situ ou repetidos tromboembolismos, atrofia com dilatação e ruptura de vasos e até necrose fibrinóide da parede vascular ${ }^{23}$. Estes achados histológicos variarão em grau e combinação segundo a causa e o tempo de evolução da HP.

\section{REFERÊNCIAS}

1. McGoon M. Screening, Early Detection, and Diagnosis of Pulmonary Arterial Hypertension: ACCP Evidence-Based Clinical Practice Guidelines. Chest 2004;126: 14S-34S

2. Guimarães JI, Lopes AA. Diagnóstico, Avaliação e Terapêutica da Hipertensão Pulmonar. Diretrizes da Sociedade Brasileira de Cardiologia. Arq Bras Cardiol 2003; 81(SVIII).

3. Himelman RB. Improved recognition of cor pulmonale in patients with severe chronic obstructive pulmonary disease. Am J Med 1988;84:891.

4. Berger M. Quantitative assessment of pulmonary hypertension in patients with tricuspid regurgitation using continuous wave doppler ultrasound. J Am Coll Cardiol 1985;6:359.

5. Fishman AJ. Perfusion lung scans vs pulmonary angiography in evaluation of suspected primary pulmonary hypertension. Chest 1983;84:679.

6. Ryan KL. Perfusion scan findings understate the severity of angiographic and hemodynamic compromise in chronic thromboembolic pulmonary hypertension. Chest 1988;93:1180.
Na HP tromboembólica crônica, resultado de obstruções sucessivas no leito vascular pulmonar, são achados comuns o comprometimento das grandes artérias elásticas ${ }^{23}$ por trombos em organização ou trombos recanalizados, como observado no caso, sendo potencialmente curável com a cirurgia de tromboendarterectomia pulmonar ${ }^{24}$. No entanto, é difícil o diagnóstico diferencial desta com hipertensão pulmonar primária, quando envolvendo pequenas artérias pulmonares. Mas como padrão geral, na HP tromboembólica crônica temos menor hipertrofia da túnica média e com muito menor freqüência lesões plexiformes, bastante freqüentes na HP primária junto com a presença de trombos e lesões trombóticas. Desta forma, o padrão morfológico observado no caso favoreceu o diagnóstico de tromboembolismo de repetição.

0 termo tromboembolismo é devido à dificuldade de distinção histológica entre o que pode ter vindo ao pulmão como êmbolo e passou a ser organizado na luz vascular, do que pode ter sido formado primariamente como trombo já na própria parede do vaso pulmonar por causa desconhecida.

Aspecto interessante neste caso é a presença de aneurisma do átrio direito. Este é achado incomum, podendo ser congênito ou secundário a trauma e está descrito em pacientes que podem ser assintomáticos, ou apresentarem arritmia cardíaca atrial ou ainda repetidos fenômenos embólicos pulmonares ${ }^{25}$. Neste caso, o aneurisma pode representar a causa primária para os achados tromboembólicos pulmonares que levaram à hipertensão pulmonar e conseqüentemente ao cor pulmonale crônico, que progrediu para insuficiência cardíaca. Esta última pode ainda ter favorecido a ocorrência de tromboses venosas, que possivelmente colaboraram para a manutenção do tromboembolismo pulmonar crônico.

(Jussara Bianchi Castelli, Léa Maria Macruz Ferreira Demarchi)

7. Fedullo PF. Chronic thromboembolic pulmonary hypertension. N Engl J Med 2001;345:1465.

8. Pengo V. Incidence of chronic thromboembolic pulmonary hypertension after pulmonary embolism. N Engl J Med 2004;350:2257.

9. Fedullo PF. The natural history acute and chronic thromboembolic disease: the search for the missing link. Eur Resp J 2000;15:435.

10. Bertina RM. Genetic approach to thrombophilia. Thromb Haemost $2001 ; 86: 92$.

11. Mateo J. Laboratory evaluation and clinical characteristics of 2132 consecutive unselected patients with venous thromboembolism results of the Spanish multicentric Study on Trombophilia (EMET Study). Thromb Haemost 1997;77:444.

12. Bombeli T. In patients symptomatic for deep-vein thrombosis factor VIII elevation is found twice as frequent as in patients symptomatic for pulmonary embolism. Thromb Haemost 2003;89:198.

13. Bonderman D. High prevalence of elevated clotting factor VIII in chronic thromboembolic pulmonary hypertension. Thromb Haemost 2003;90:372. 
14. Sorensen HT. Prognosis of cancers associated with venous thromboembolism. N Engl J Med 2000;343:1846.

15. Ray, JG. Meta-analysis of hyperhomocysteinemias as a risk factor for thromboembolic disease. Arch Intern Med 1998;158:2101.

16. Galli M. Lupus anticoagulants are stronger risk factors for thrombosis than anticardiolipin antibodies in the antiphospholipid syndrome: a systematic review of the literature. Blood 2003;101:1827.

17. Riedel M. Longterm follow-up of patients with thromboembolism: late prognosis and evolution of hemodinamic and respiratory data. Chest $1982 ; 81: 151$

18. Long term domiciliary oxygen therapy in chronic hypoxic cor pulmonale complicating chronic bronchitis and emphysema. Report of the Medical Research Council Working Party. Lancet 1981;1:681.

19. Roberts $\mathrm{DH}$. Oxygen therapy improves cardiac index and pulmonary vascular resistance in patients with pulmonary hypertension. Chest $2001 ; 120: 1547-55$

20. Califf RM. A randomized controlled trial of epoprostenol therapy for severe congestive heart failure: The Folan International Randomized Survival Trial (FIRST). Am Heart J 1997;134:44.

21. Olschewski H, Simonneau G, Galie N et al. Inhaled iloprost for severe pulmonary hypertension. N Engl J Med 2002;347:322-9.

22. Jatene FB. Tratamento cirúrgico da hipertensão pulmonar tromboembólica. Rev Soc Cardiol Estado de São Paulo 2000;5:640.

23. Galie N, Torbicki A, Barst R et al. Grupo de Trabajo sobre el diagnostico y tratamiento de la Hipertension Arterial Pulmonar de la Sociedad Europea de Cardiologia. Guidelines on diagnosis and treatment of pulmonary arterial hypertension. The Task Force on Diagnosis and Treatment of Pulmonary Arterial Hypertension of the European Society of Cardiology. Eur Heart J 2004;25(24):2243-78.

24. Fedullo PF, Auger WR, Channick RN, Moser KM, Jamieson SW. Chronic thromboembolic pulmonary hypertension. Clin Chest Med. 1995;16(2):353-74.

25. Barberato SH, Barberato MF, Avila BM, Perretto S, Blume Ld Ldo R, Chamma Neto M. Aneurysm of the Right Atrial Appendage. Arq Bras Cardiol 2002;78(2):239-41. 